\title{
Superposition and higher-order spacing ratios in random matrix theory with application to complex systems
}

\author{
Udaysinh T. Bhosale \\ Department of Physics, National Institute of Technology, Nagpur 440010, India and \\ Physical Research Laboratory, Navrangpura, Ahmedabad 380009, India
}

(Dated: August 9, 2021)

\begin{abstract}
The statistical properties of spectra of quantum systems within the framework of random matrix theory is widely used in many areas of physics. These properties are affected, if two or more sets of spectra are superposed, resulting from the discrete symmetries present in the system. Superposition of spectra of $m$ such circular orthogonal, unitary and symplectic ensembles are studied numerically using higher-order spacing ratios. For given $m$ and the Dyson index $\beta$, the modified index $\beta^{\prime}$ is tabulated whose nearest neighbor spacing distribution is identical to that of $k$-th order spacing ratio. For the case of $m=2(m=3)$ in COE (CUE) a scaling relation between $\beta^{\prime}$ and $k$ is given. For COE, it is conjectured that for $k=m+1(m \geq 2)$ and $k=m-3$-th $(m \geq 5)$ order spacing ratio distribution the $\beta^{\prime}$ is $m+2$ and $m-4$ respectively. Whereas in the case of CSE, for $k=m+1$ $(m \geq 2)$ and $k=m-1$-th $(m \geq 3)$ the $\beta^{\prime}$ is $2 m+3$ and $2(m-2)$ respectively. We also conjecture that for given $m(k)$ and $\beta$, the sequence of $\beta^{\prime}$ as a function of $k(m)$ is unique. Strong numerical evidence in support of these results is presented. These results are tested on complex systems like the measured nuclear resonances, quantum chaotic kicked top and spin chains.
\end{abstract}

\section{INTRODUCTION}

Random matrix theory (RMT) has been successfully applied to study the spectral fluctuations in various complex quantum systems [1 9]. These include spin chains from condensed matter physics [10 14], nuclear physics [1, 2, 15], chaotic billiards [16, 17], etc. These fluctuations are used to characterize various phases of these systems, for example, thermal or localized phase of spin chains 12 14, 18], integrable to chaotic limit of the underlying classical system [19, 20], etc. For correct characterization of the system, its spectra needs to be desymmetrized [21]. If the Hamiltonian $H$ for given system an additional symmetry $S$, i.e. $[H, \hat{S}]=0$, where $\hat{S}$ is the operator corresponding to $S$ then the eigenvalues gets superposed. This can lead to entirely different fluctuation properties failing to characterize the system [8, 21 27].

Due to symmetry $S$ the $H$ becomes block diagonal in the basis formed by the eigenfunctions of $S$, i.e., $H=H_{1} \oplus H_{2} \oplus \ldots \oplus H_{m}$. Here, $m$ denotes the number of non-degenerate eigenvalues of $S$. Thus, due to the symmetry $S$, in the spectra of $H$, the eigenvalues from different blocks get superposed. Symmetries also have played an important role in our understanding of many areas of physics [28 31], mathematics [31, 32], biology [33], etc. The importance of symmetries can be understood from the works of Emmy Noether, where she has related continuous symmetry and conservation laws in

*Electronic address: udaybhosale0786@gmail.com her famous theorem [34, 35].

Symmetries have also played an important role in the RMT [7, 8]. This goes back to Wigner who defined a class of Gaussian random matrix ensembles to understand the fluctuations in the nuclear spectra. The class of ensemble one uses depends on the symmetry present in the system. In RMT, the spectral fluctuations are modeled using the most popular measure namely the nearest neighbour (NN) level spacings, $s_{i}=E_{i+1}-E_{i}$, where $E_{i}, i=1,2, \ldots$ are the eigenvalues of the Hamiltonian $H$. Wigner surmised that in time-reversal invariant systems without a spin degree of freedom, these spacings are distributed as $P(s)=(\pi / 2) s \exp \left(-\pi s^{2} / 4\right)$, which indicates the level repulsion. For these systems, the statistical properties of the spectra are modelled correctly by the Gaussian Orthogonal Ensemble (GOE) having Dyson index $\beta=1$. Other ensembles that are used commonly in RMT are Gaussian unitary ensemble (GUE) and Gaussian symplectic ensemble (GSE) having Dyson index $\beta=2$ and 4 respectively having applications to various fields [4, 36]. In this work, the circular class of ensembles has been studied [7] and based on previous studies our results can be extended to Gaussian ensembles for large matrix dimensions under certain condition as explained in Sec II 7, 8, 21, 37 39]. The symmetries that are used in defining respective Gaussian ensembles are the same for those of circular ensembles. Indices $\beta=1$, 2 and 4 corresponds to Dyson's threefold way and have played an important role in physics. Matrix representation for these indices was given in the initial development of RMT. But these ensembles are valid and exits for continuous parameter $\beta \in(0, \infty)$ and a tridiagonal random matrix model have been defined for them [40]. It has been used recently in the study of level statistics of many-body localization for $\beta \in(0,1]$ [41, 42]. The index 
$\beta$ is interpreted as the inverse temperature of $T=1 / \beta$ in the RMT literature.

In 1984, Bohigas, Giannoni, and Schmidt conjectured that quantum chaotic systems display NN level statistics consistent with that of an appropriately chosen random matrix ensemble 9, 16]. This conjecture is supported by many theoretical studies [43-45]. Due to the additional symmetry $S$, which may not be known apriori, the eigenvalues from different blocks get superposed. This results in level clustering of $\mathrm{NN}$ and one obtains their spacings distribution to be Poissonian [8, 9, 46 48], $P(s)=\exp (-s)$, which also corresponds to the NN spectral fluctuations of integrable systems known as Berry and Tabor's conjecture [49]. This implies that to study the genuine spectral correlations, eigenvalues must be drawn from the same subspace.

Motivated by the works of Wigner, Dyson introduced a new class of ensembles of random matrices known as circular $\beta$-ensembles which are measures in the spaces of unitary matrices [50]. They have played important roles in RMT. The Dyson index $\beta=1,2$ and 4 corresponds to Circular Orthogonal Ensemble (COE), Circular Unitary Ensemble (CUE) and Circular Symplectic Ensemble (CSE) respectively. These ensembles have found applications in the scattering from a disordered cavity [7], condensed matter and optical physics [4]. The algorithm for generating these ensembles numerically is non-trivial compared to that of Gaussian ensembles and is given in Ref. [51]. Similar to Gaussian $\beta$-ensemble, the circular $\beta$-ensemble is also defined for continuous parameter $\beta \in(0, \infty)$ and a corresponding tridiagonal model is defined for them [24, 52].

Previous studies have shown connections within ensembles corresponding to $\beta=1,2$ and 4 . A theorem which relates the properties of the CUE and COE has been conjectured in Ref. 22 and later proved by Gunson [23]. It states that the alternate eigenvalues obtained after superposition of spectra of two matrices ( $m=2$ as per our notation) of the same dimension from COE belong to that of CUE. A similar theorem relating properties of $\mathrm{COE}$ and CSE were proved in Ref. [53]. It states that the alternate eigenvalues of an even-dimensional COE belong to that of CSE. Thus, these two theorems together state that all the statistical properties of the three ensembles are derivable from that of $\mathrm{COE}$ alone [53]. In fact, these two theorems hold at the level of joint probability distribution function (jpdf). As a corollary of these theorems, one can also say that level fluctuations of CUE and CSE can be obtained using COE. It is also conjectured that similar relationships hold true for Gaussian ensembles of infinite dimensions, in which the GOE underlies the GUE and GSE [38, 39].

There are recent studies where higher-order spacing ratios are studied in the superposed spectra [21]. There it is shown that when $m$ number of COE spectra are superposed then the distribution of the $m$-th order spacing ratios is same as that of NN spacing ratios of the circular ensemble with modified Dyson index $m$. (The definition of higher-order spacing ratios will be given in detail in Sec III). This result is then used to find symmetries in various physical systems like spin chains, quantum billiards and experimentally measured nuclear resonances. Similarly in Ref. 25] the distribution of NN spacing as well as NN spacings ratio is studied in Gaussian ensembles when discrete symmetries are present with no restriction of their numbers. These results are then applied to quantum many-body systems, anyonic chains to periodically-driven spin systems and quantum clock models. It can be seen that only the special cases of spacings and their ratios for given $m$ are studied in Ref. 21, 25]. In this paper, our main aim is to study $k$-th higher-order spacing ratios for given $m$ superpositions for each of the COE, CUE and CSE ensembles. There will be no restriction on the value of $k$ as it was in Ref.[21] where $k=m$. We will be validating our $\mathrm{COE}$ results by testing them on the physical model like the quantum kicked top (QKT), experimentally measured nuclear resonances and spin Hamiltonian. Our results can also be used as a stringent test for studying symmetries in other systems.

The structure of the paper is as follows: In Sec Idefinition of various quantities, namely, the NN spacing ratios, higher-order spacing ratios are given. Previous studies from random matrix theory and other fields using these definitions are presented. In Sec III our results using the higher-order spacing ratios of superposition of COEs are presented. In Sec IV we have shown the application of these results to the physical systems. In Sec V (Sec VI) we have presented results on higher-order spacing ratios of superposition of CUEs (CSEs). In SecVII various numerical methods used in this paper in support of our results are presented. In Sec VIII summary of the results and conclusion is given.

\section{PRELIMINARIES}

For the study of the spacing distribution, one needs to unfold the spectra which removes the system dependent spectral features, i.e., the average part of the density of states (DOS) [3, 6, 8, 49, 54]. This procedure is nonunique and cumbersome in many cases which can give misleading results [55]. This difficulty can be solved by using the NN spacing ratios [56], i.e., $r_{i}=s_{i+1} / s_{i}, i=1$, $2, \ldots$, since it is independent of the local DOS and thus does not require unfolding. The distribution of $r_{i}, P(r)$ has been obtained for Gaussian ensembles and is given as follows [57, 58]:

$$
P(r, \beta)=\frac{1}{Z_{\beta}} \frac{\left(r+r^{2}\right)^{\beta}}{\left(1+r+r^{2}\right)^{(1+3 \beta / 2)}}, \quad \beta=1,2,4
$$

where $Z_{\beta}$ is the normalization constant that depends on $\beta$. This quantity has found many applications, like numerical investigation of many-body localization [41, 56, 59 63], localization in constrained quantum system [64], quantifying the distance from integrability on 


\begin{tabular}{|c|c|c|c|c|c|c|c|}
\hline$k$ & $\beta=1$ & $\beta=2$ & $\beta=3$ & $\beta=4$ & $\beta=5$ & $\beta=6$ & $\beta=7$ \\
\hline 1 & 1 & 2 & 3 & 4 & 5 & 6 & 7 \\
2 & 4 & 7 & 10 & 13 & 16 & 19 & 22 \\
3 & 8 & 14 & 20 & 26 & 32 & 38 & 44 \\
4 & 13 & 23 & 33 & 43 & 53 & 63 & 73 \\
5 & 19 & 34 & 49 & 64 & 79 & 94 & 109 \\
6 & 26 & 47 & 68 & 89 & 110 & 131 & 152 \\
7 & 34 & 62 & 90 & 118 & 146 & 174 & 202 \\
8 & 43 & 79 & 115 & 151 & 187 & 223 & 259 \\
\hline
\end{tabular}

TABLE I: Tabulation of higher-order indices $\beta^{\prime}$ for various $k$ and $\beta$ using Eq. (3).

finite size lattices [26, 65 68] and to study localization transition in Lévy matrices [69], to study symmetries in various complex systems [21, 25], to study degree of chaoticity in different random matrix models 70 , to study quantum chaos in Sachdev-Ye-Kitaev models 71 73] and quantum field theory 74. Variations of the spacing ratios have been studied in the recent past [58, 75-77] including generalization to complex eigenvalues [78]. In this work, the non-overlapping $k$-th order spacing ratio is considered, where no eigenvalue is shared between the spacings of numerator and denominator, defined as follows:

$$
r_{i}^{(k)}=\frac{s_{i+k}^{(k)}}{s_{i}^{(k)}}=\frac{E_{i+2 k}-E_{i+k}}{E_{i+k}-E_{i}}, \quad i, k=1,2,3, \ldots
$$

This ratio has been used to study higher-order fluctuation statistics in the Gaussian [79], circular [79] and Wishart ensembles [80], and a scaling relation is given as follows:

$$
\begin{aligned}
P^{k}(r, \beta, m=1) & =P\left(r, \beta^{\prime}\right), \quad \beta \geq 1 \\
\beta^{\prime} & =\frac{k(k+1)}{2} \beta+(k-1), \quad k \geq 1 .
\end{aligned}
$$

It tells that the distribution of $k$-th order spacing ratio for a given $\beta$ ensemble is same as that of NN spacing ratios of $\beta^{\prime}(>\beta)$ ensemble. It has been applied successfully to various physical systems like spin chains, chaotic billiards, Floquet systems, observed stock market, etc. 79 81]. It is also used recently to find the symmetries in complex systems [21].

It should be noted here that the results obtained for the case $k=1$ are found to be universal since it does not depend on the local DOS. Whereas for $k>1$ one needs to be careful since the DOS, which changes from ensemble to ensemble, can affect the distribution of $r_{i}^{(k)}$. For example, in the case of circular ensembles (introduced in Sec III) the DOS is uniform, for Gaussian ensembles it is Wigner's semicircle whereas for Wishart ensemble it is given by Marchenko-Pastur distribution [8]. Thus, only in the case of circular ensembles DOS will not affect the higher-order spacing ratios. In the case of physical systems DOS can be different even if their NN fluctuation properties are explained by the same kind of RMT ensemble 2, 12 14, 26, 27, 37, 82. Thus, the results for $k>1$ can not be claimed to be universal that easily. But evidences from Ref. 37] (see Fig.5 therein) suggest that for given $k$ if the matrix dimension is increased large enough, which will depend on the RMT ensemble and the physical system under consideration, then our RMT results can be applied to them. This means that the effects of nonuniform density can be minimised by increasing the matrix dimension for given value of $k$. This will also be demonstrated in SecIV where we apply the RMT results to the physical system of spin chain.

Recently, same relation between the higher order and the NN spacing distributions had been shown rigorously which is tested on random spin systems and non-trivial zeros of Riemann zeta function [39, 83 87]. In Ref. 21] (as explained in the Introduction) the distribution of the $m$-th order spacing ratios after superposing the spectra of $m$ COEs is studied. It is shown to be converging to the distribution of the $\mathrm{NN}$ spacing ratios $P\left(r, \beta^{\prime}\right)$ with $\beta^{\prime}=m$ i.e.

$$
P^{k}(r, 1, m)=P\left(r, \beta^{\prime}\right) \text {, where } \beta^{\prime}=k=m .
$$

The Eq. (3) is tabulated for few values of $\beta$ and $k$ in Table I. It can be observed from the $\beta=1$ series in Table \ that the $\beta=4$ series appears at its even places. This is because of the relation between COE and CSE exists at the level of the jpdf of the eigenvalues 22, 23] as discussed in the introduction. This observation plays an important role in further analysis in the subsequent part of this paper. The special case of the Eq. (3) for $0 \leq \beta \leq 1$ is given in Refs. [88, 89] at the level of the joint probability distribution of eigenvalues. There, it is shown that the jpdf of every $k$-th eigenvalue in certain $\beta$-ensembles with $\beta=2 / k$ is equal to that of another $\beta$ ensemble with $\beta=2 k$. Based on numerical simulations the results of our work will now be presented.

\section{SUPERPOSITION AND HIGHER-ORDER SPACING RATIOS IN COE}

In this work, the main object of study is the circular $\beta$-ensembles. The jpdf is given as follows:

$$
Q_{N, \beta}\left[\left\{\theta_{i}\right\}\right]=C_{N, \beta} \prod_{k>j}^{N}\left|\exp \left(i \theta_{j}\right)-\exp \left(i \theta_{k}\right)\right|^{\beta}
$$

where $N$ is the dimension and $C_{\beta, N}=(2 \pi)^{-N}\{\Gamma(1+$ $\beta / 2)\}^{N}\{\Gamma(1+N \beta / 2)\}^{-1}$ is the normalization constant [7, 8]. The eigenvalues $\theta_{i}$ are distributed uniformally on the unit circle and display level repulsion, characterized by $\beta \geq 0$ [8]. Larger the value of $\beta$ larger is the repulsion. It can be seen that if $\beta=0$ is put in the jpdf all the eigenvalues are independent and thue uncorrelated. For such eigenvalues the level statistics follows a Poisson law.

In this section, we consider the superposition of $m \geq 2$ number of COEs and study its non overlapping $k$-th order 
spacing ratio distribution $P^{k}(r, \beta, m)$. For $\mathrm{COE}, \beta=1$ is taken and is used in modeling Hamiltonians which possess time-reversal symmetry and no half-integer spin [8]. This is then related with the NN spacing ratio distribution $P\left(r, \beta^{\prime}\right)$ for $\beta^{\prime}>\beta$. For given $m$ and $k$ we tabulate the value of $\beta^{\prime}$ for which both these distributions are very close to each other numerically. These values are given in Table II for $m=2$ to 7 and various values of $k$. It can be seen that except few all values of $\beta^{\prime}$ are whole number (this is the case for the superposition in CUEs and CSEs, which will be discussed in subsequent parts of the paper). These results are plotted in Figs. 1, 2, 3, 4 and 5. The insets shows the $D\left(\beta^{\prime}\right)$ function, the minimum of which gives the best fit $P\left(r, \beta^{\prime}\right)$ to the numerical data $P^{k}(r, \beta, m)$. Its detailed definition and other numerical methods with which we find $\beta^{\prime}$ will be discussed in Section VII. We have also plotted a representative data for

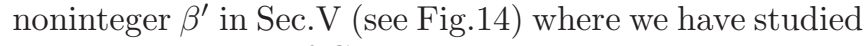
the superposition of CUEs.

The $m=2$ is an interesting case for which we have obtained scaling relations for even and odd values of $k$ and $\beta^{\prime}$ as given below:

$$
\beta^{\prime}=\frac{5 k}{2}-3+\frac{(k-2)(k-4)}{4}, \quad k=2,4,6, \ldots
$$

and

$$
\beta^{\prime}=3 k-5+\frac{(k-3)(k-5)}{4}, \quad k=3,5,7, \ldots
$$

For even $k$ the scaling relation reduces to Eq. (3) for $\beta=2$ by suitable change of variables as $l=k / 2$ where $l=1,2,3, \ldots$. This is a known result on the connection between CUE and superposition of two COEs [22, 23]. For odd $k$ changing the variable as $q=(k-1) / 2$ then Eq. 7 reduces to a simpler form as $\beta^{\prime}=q(q+3)$ for $q=1,2,3 \ldots$. It can be compared with Eq. (3) and can be seen that it does not reduces to Eq. (3) for any $\beta$. Thus, using the scaling relation in Eq. (7), no statement can be made at the level of jpdf of circular $\beta$-ensemble.

Based on the results in Table III we have given two conjectures on the lines of Eq. (44) (see Ref. 21]) at the level of spectral fluctuations. The first conjecture is as follows:

$$
P^{k}(r, 1, m)=P\left(r, \beta^{\prime}\right), \text { for } \beta^{\prime}=k+1=m+2
$$

and $m \geq 2$, while the second one is as follows:

$$
P^{k}(r, 1, m)=P\left(r, \beta^{\prime}\right), \text { for } \beta^{\prime}=k-1=m-4
$$

and $m \geq 5$. Our conjectures hold true for asymptotic value of $N$. These conjectures have appeared in our arXiv preprint [90].

\section{TESTING RMT RESULTS TO PHYSICAL SYSTEMS}

In this section, we test our COE results on the physical model of the quantum kicked top (QKT), experimentally

\begin{tabular}{|c|c|c|c|c|c|c|}
\hline$k$ & $\begin{array}{c}m=2 \\
\beta^{\prime}\end{array}$ & $\begin{array}{c}m=3 \\
\beta^{\prime}\end{array}$ & $\begin{array}{c}m=4 \\
\beta^{\prime}\end{array}$ & $\begin{array}{c}m=5 \\
\beta^{\prime}\end{array}$ & $\begin{array}{c}m=6 \\
\beta^{\prime}\end{array}$ & $\begin{array}{c}m=7 \\
\beta^{\prime}\end{array}$ \\
\hline 1 & 0 & 0 & 0 & 0 & 0 & 0 \\
2 & 2 & 1.25 & 1 & 1 & 0.75 & 0.75 \\
3 & 4 & 3 & 2.5 & 2 & 2 & 2 \\
4 & 7 & 5 & 4 & 3.5 & 3 & 3 \\
5 & 10 & 7.5 & 6 & 5 & 5 & 4 \\
6 & 14 & 10 & 8 & 7 & 6 & 6 \\
7 & 18 & 13 & 11 & 9 & 8 & 7 \\
8 & 23 & 17 & 13 & 12 & 10 & 9 \\
9 & 28 & 20 & 16 & 14 & 12 & 11 \\
10 & 34 & 24 & 20 & 17 & 15 & 13 \\
11 & 40 & 29 & 23 & 20 & 17 & 16 \\
12 & 47 & 33 & 27 & 23 & 20 & 18 \\
13 & 54 & 38 & 31 & 26 & 23 & 21 \\
14 & & 43 & 35 & 30 & 26 & 23 \\
15 & & 49 & 39 & 33 & 29 & 26 \\
16 & & & 44 & & 32 & 29 \\
17 & & & & & & 32 \\
18 & & & & & & 35 \\
19 & & & & & & 39 \\
20 & & & & & & 42 \\
\hline
\end{tabular}

TABLE II: Tabulation of higher-order indices $\beta^{\prime}$ for various $k$ and superposition of $m$ COEs each having dimension $N=$ 8400 .

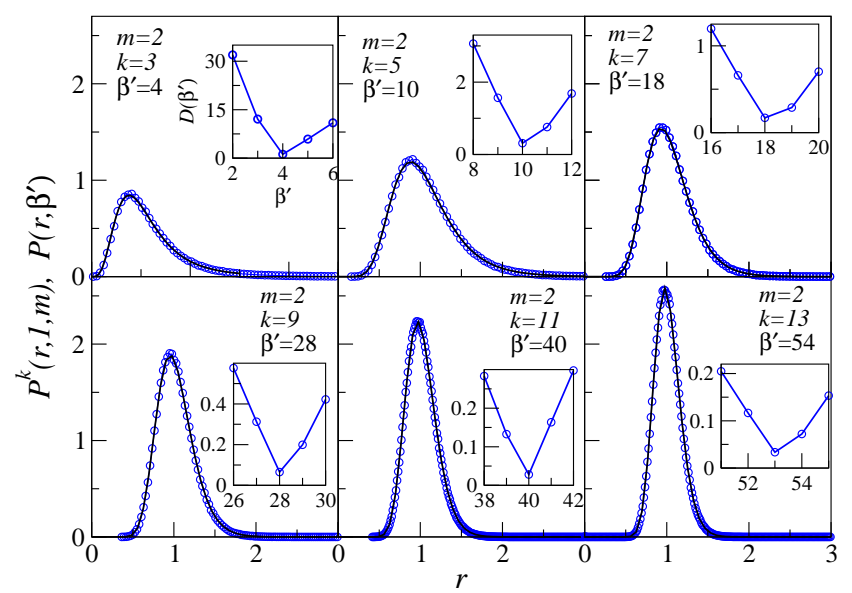

FIG. 1: (Color online) Distribution of the $k$-th order spacing ratios (circles) for a superposition of $m=2 \mathrm{COE}$ spectra. The dimension of the matrices is $N=8400$. The solid curve corresponds to $P\left(r, \beta^{\prime}\right)$ as given in Eq. (1) with $\beta^{\prime}$ given in Table The insets shows $D$ as a function of $\beta^{\prime}$.

measured data of nuclear resonances and a spin Hamiltonian. Using these systems it will be shown that our results hold true for $m=2$ case. First, we consider the model of QKT. This is a fundamental and important time-dependent model for the chaotic Hamiltonian system. This model was introduced in [19] and has been the topic of theoretical and experimental research since then [19, 191 106]. It has been realized in various experiments, namely, the hyperfine states of cold atoms [95], three coupled superconducting qubits [98] and in a two- 


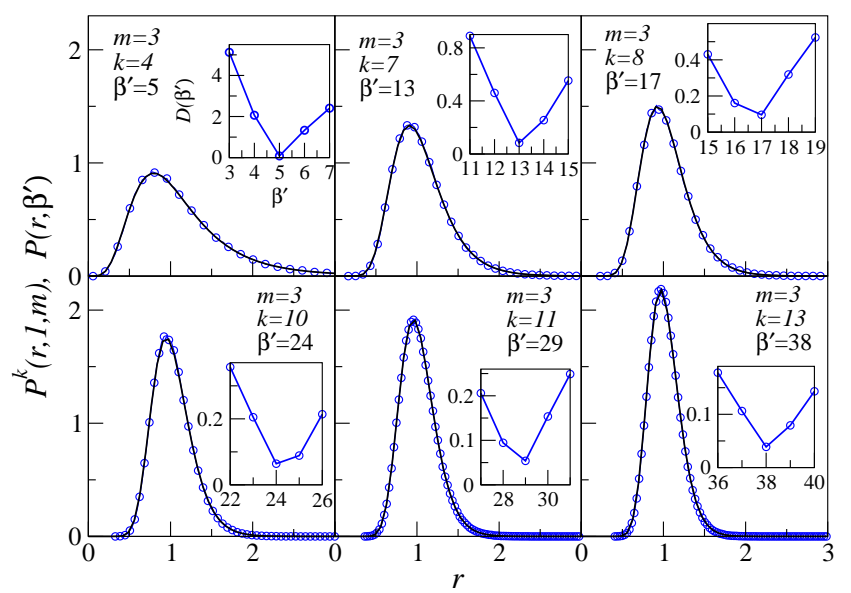

FIG. 2: (Color online) Same as Fig 1 for $m=3$.

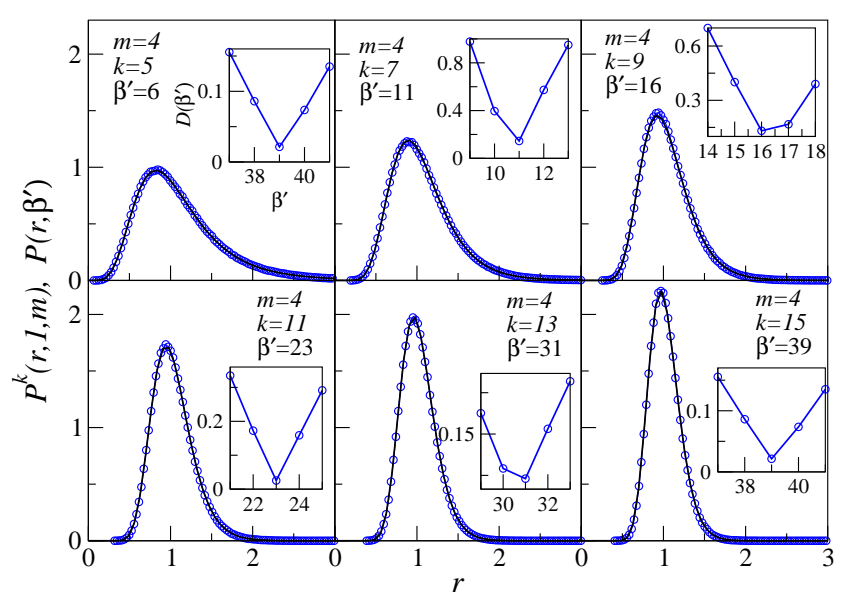

FIG. 3: (Color online) Same as Fig 1 for $m=4$.

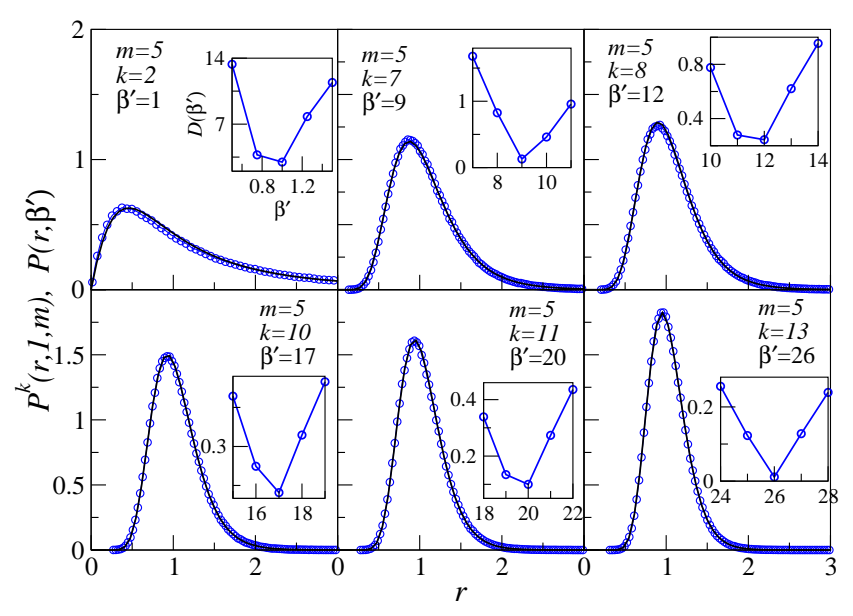

FIG. 4: (Color online) Same as Fig 1 for $m=5$.

qubit NMR system [102]. This is also an important model from the perspective of random matrix theory and quan-

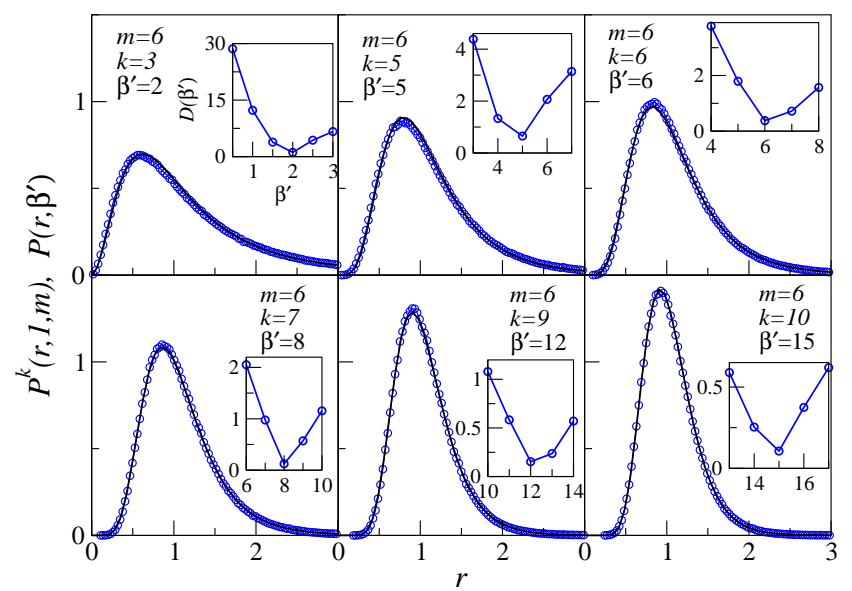

FIG. 5: (Color online) Same as Fig 1 for $m=6$.

tum information. This model shows regular to chaotic behavior as a function of chaoticity parameter. Effects of the underlying phase space on various measures quantum correlations are studied [98 100, 107]. For classical limit being fully chaotic, the NN fluctuations of symmetry reduced spectra of the QKT corresponds to that of COE ensemble [16, 19].

QKT is characterized by an angular momentum vector $\mathbf{J}=\left(J_{x}, J_{y}, J_{z}\right)$ and its components obey the standard algebra of angular momentum. The unitary time evolution operator for QKT is given as follows:

$$
\widehat{U}=\exp \left(-i p J_{y}\right) \exp \left(-i \frac{k}{2 j} J_{z}^{2}\right)
$$

It represents free precession of the top around $y$ axis with angular frequency $p$ while the second term is periodic $\delta$ kicks applied to the top. Here, $k$ is called as the kick strength or chaos parameter. For $k=0$ the top is integrable and for $k>0$ it becomes increasingly chaotic. For given $j$ the Hilbert space dimension is equal to $2 j+1$.

As discussed in Ref.[19], for $p \neq \pi / 2$, the case relevant for us, there are two symmetries present in QKT, since $\widehat{U}$ commutes with the rotation operator $\hat{R}_{y}$ having two eigenvalues. Thus, the matrix representation of $\widehat{U}$ in the basis of $R_{y}$ is block diagonal consisting of two blocks and their dimensions are $j$ and $j+1$. The large $j$ case is relevant of us as these dimensions are very close to each other. For the fully chaotic case, the eigenvalue fluctuations of $\widehat{U}$ in each such block is found to follow COE statistics [19]. Taking these eigenvalues together and studying their higher-order spacings ratio is an ideal case for our study. We can compare them with our COE results of $m=2$.

For our study $j=1000$ and twenty realizations for large and different values of $k$ is taken. Thus, the dimension of the matrix $\widehat{U}$ is 2001. The dimension of two blocks when $\widehat{U}$ is written in the eigenbasis of $\hat{R}_{y}$ is 1000 and 1001 respectively. Thus, we can test our RMT re- 
sults of $m=2$ case of COE. The results are plotted in Figs. 6 and 7 for $k=2$ to 13 . It can be seen here that the results agree very well with the RMT results, $m=2$ case of COE in Table III thus implying that there are two symmetries in the QKT which we were already known apriori [19]. The RMT results hold true for such a large value of $k$ due to the uniform density in both, the COE ensemble and the QKT (as discussed in the Sec III).

Now, we go on to test our results to experimentally measured nuclear resonances of Tantalum $\left(\mathrm{Ta}^{181}\right)$ 82, 108]. It is known that it belongs to the GOE and there are two symmetries present in it [82, 108]. We have 434 such resonances and the results are plotted in Fig. (8). The value of $\beta^{\prime}$ is chosen such that $P\left(r, \beta^{\prime}\right)$ is best fit to the data. The results are compared with $m=2$ case of COE in Table III It can be seen that the results hold true only for $k=2$ and 3 . From $k=4$ onwards we observe deviations from our RMT results. This is due to the nonuniversal effects in the DOS (not shown here) and the small sample size. With this example, we have tested our COE result on a GOE system with small sample size and found the number of symmetries in it successfully.

Now, a Hamiltonian corresponding to spin- $1 / 2$ chain [27, 109] is considered as follows:

$$
\begin{aligned}
H= & \sum_{i=1}^{L-1}\left[J_{x y}\left(S_{i}^{x} S_{i+1}^{x}+S_{i}^{y} S_{i+1}^{y}\right)+J_{z} S_{i}^{z} S_{i+1}^{z}\right] \\
& +\alpha \sum_{i=1}^{L-2}\left[J_{x y}^{\prime}\left(S_{i}^{x} S_{i+2}^{x}+S_{i}^{y} S_{i+2}^{y}\right)+J_{z}^{\prime} S_{i}^{z} S_{i+2}^{z}\right]
\end{aligned}
$$

where $L$ is total number of sites, the NN coupling strengths in three directions are denoted by $J_{x y}$ and $J_{z}$ (couplings in $x$ and $y$ directions are same). Similarly, $J_{x y}^{\prime}$ and $J_{z}^{\prime}$ are the next-NN coupling strengths. For $\alpha=0$ this Hamiltonian is integrable 21]. Whereas it is chaotic for $\alpha \gtrsim 0.2$ and follows GOE statistics 27]. There are various symmetries in this model [110, 111]. The first one is due to conservation of total spin in the $z$ direction denoted as $S_{z}=\sum_{i=1}^{L} S_{i}^{z}$. For our work we are restricting to the case $S^{z}=0$ (even $L$ ) and $S^{z}=-1 / 2$ (odd $L$ ) such that the block-matrix is of maximum possible dimension. The Hamiltonian commutes with the parity operator with eigenvalues \pm 1 leading to two invariant subspaces in a given $S_{z}$ block. Results for this case are plotted in Fig 9 for odd value of $L$. It can be seen that our RMT results for $m=2$ case from Tabl $\amalg$ holds true for $k=2,3$ and 4 . For even $L$, the Hamiltonian also commutes with the operator corresponding to rotation symmetry with eigenvalues \pm 1 . Thus, in this case there will be totally four invariant subspaces in a given $S_{z}$ block. The results for this case are plotted in Figs 10 and 11] In this case our results hold from $k=2$ to 6 with the corresponding RMT results for $m=4$ from Tabl@ Thus, our $m=2$ and $m=4$ the COE results agree with spin Hamiltonian having GOE statistics when there are two and four symmetries are present in it respectively. In the later case the block-matrix dimension is 3432 whereas in the previous case it is 1716 . In this case also the deviations are due to the nonuniversal effects in DOS which is Gaussian in nature (not shown here). But the results seems to improve due to the increased matrix dimension.

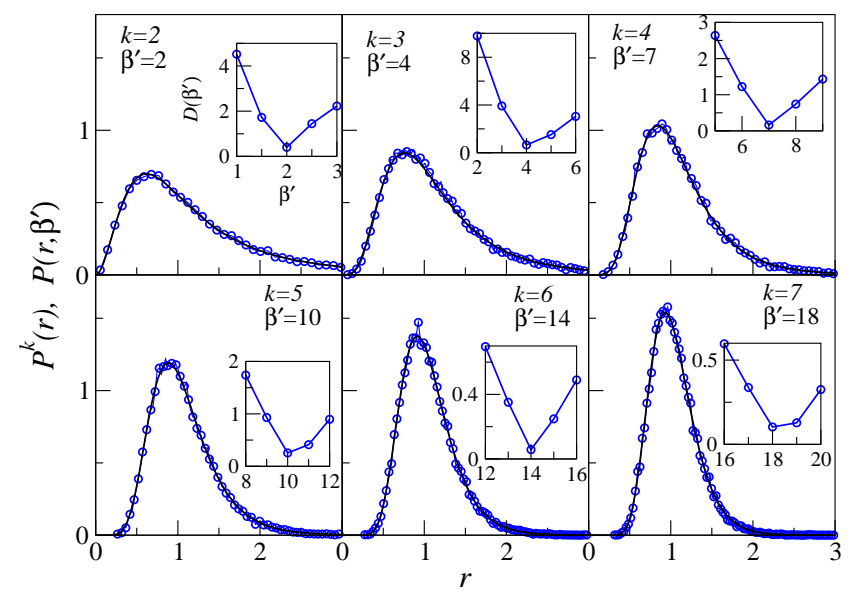

FIG. 6: (Color online) The distribution of the $k$-th order spacing ratios for $k=2$ to 7 is shown for the QKT. The numerical data $P^{k}(r)$ (circles) are obtained from the computed eigenvalues of QKT. The solid line represents $P\left(r, \beta^{\prime}\right)$, with $\beta^{\prime}=2,4,7,10,14,18$.

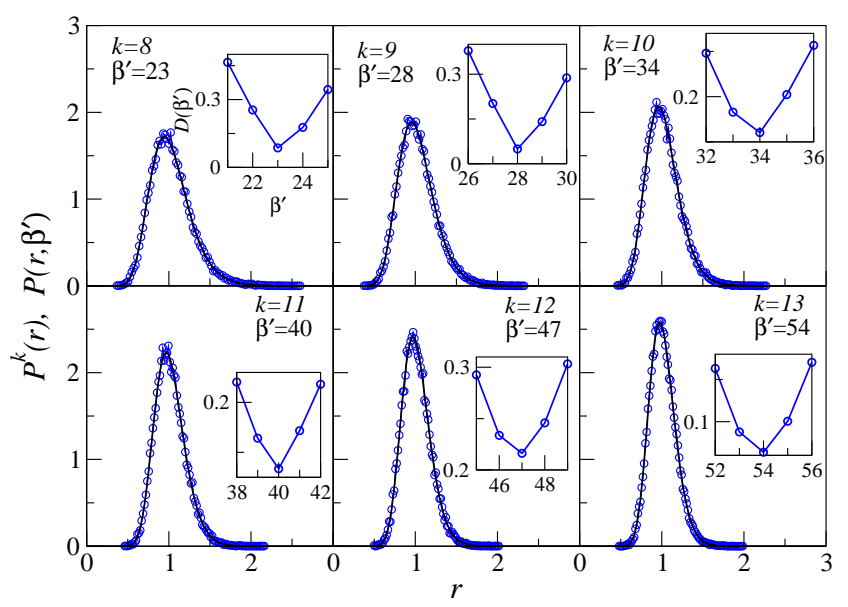

FIG. 7: (Color online) Same as Fig. 6 but for $k=8$ to 13 and $\beta^{\prime}=23,28,34,40,47,54$.

\section{SUPERPOSITION AND HIGHER-ORDER SPACING RATIOS IN CUE}

In this section, we study higher-order spacings ratio in the superposition of CUEs on the lines of Section III where superposition of COEs is studied. The CUE is used in modelling Hamiltonians which lack time-reversal 


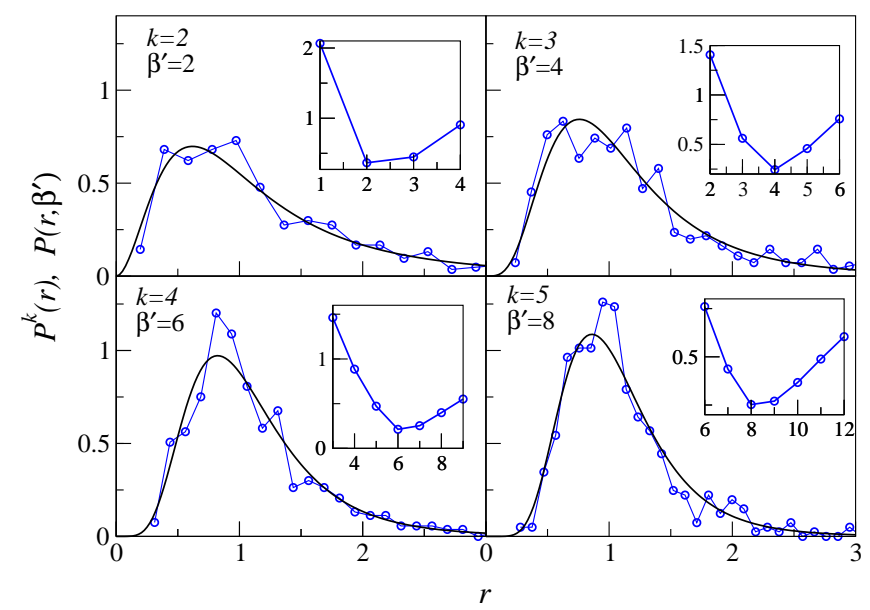

FIG. 8: (Color online) Same as Fig. 6 but for experimentally measured nuclear resonances of $\mathrm{Ta}^{181}$. Here, $k$ varies from 2 to 5 while for solid curves $\beta^{\prime}=2,4,6$ and 8 .

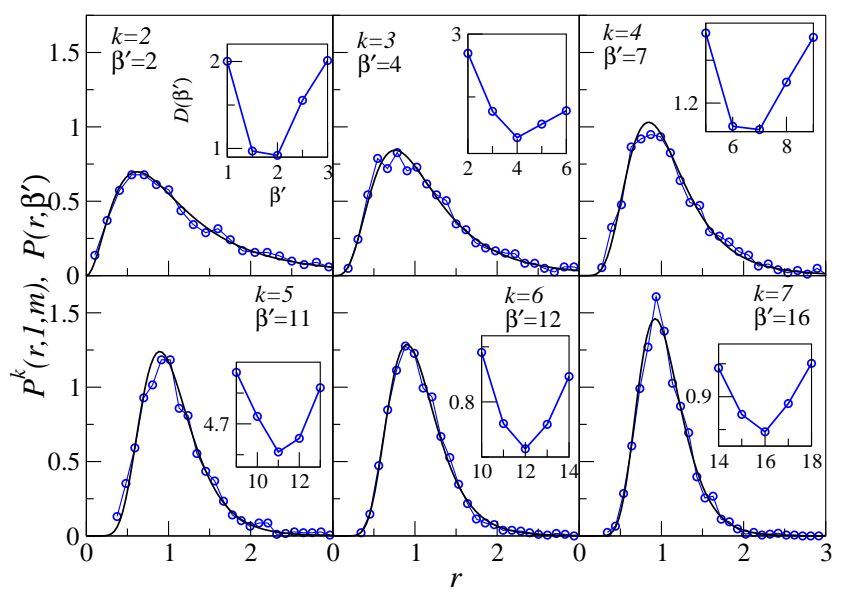

FIG. 9: (Color online) The distribution of the $k$-th order spacing ratios for $k=2$ to 7 is shown for the spin- $1 / 2$ chain Hamiltonian with $L=13$ with 7 up spins, $J_{x y}=J_{x y}^{\prime}=1$, $J_{z}=J_{z}^{\prime}=0.5$ and $\alpha=0.5$. The dimension of the blockmatrix is 1716 . The numerical data $P^{k}(r)$ (circles) are obtained from the computed eigenvalues of the Hamiltonian. The solid line represents $P\left(r, \beta^{\prime}\right)$, with $\beta^{\prime}=2,4,7,11,12,16$.

symmetry [8]. The results are tabulated in Table $\amalg$ for $m=2$ to 5 and various values of $k$. In this case also except few all values of $\beta^{\prime}$ are the whole number. The results are plotted in Figs 12,13 and 14. The Fig.14 shows noninteger values of $\beta^{\prime}$ which is found by best fit.

In the case of superposing of CUEs $m=3$ is an interesting case for which we have obtained a scaling relation for even and odd values of $k$ and $\beta^{\prime}$ as given below:

$$
\beta^{\prime}=4 k-9+\frac{(k-4)(k-6)}{4}, k=4,6,8, \ldots
$$

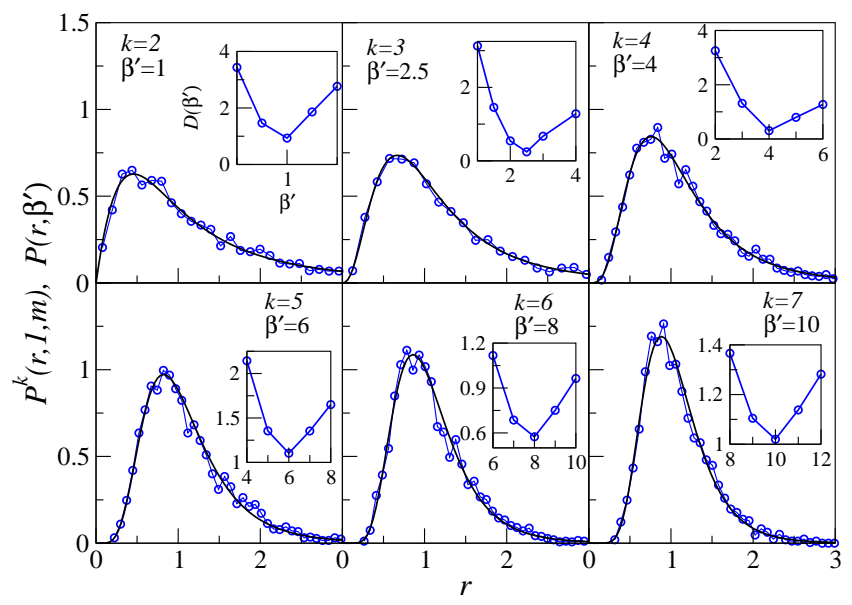

FIG. 10: (Color online) Same as Fig. 9 but for $L=14$ and $\beta^{\prime}=1,2.5,4,6,8,10$. Here 7 spins are up and the dimension of the block-matrix is 3432 .

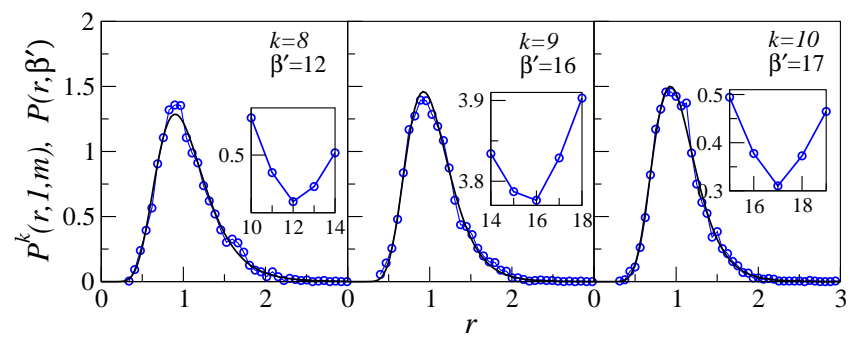

FIG. 11: (Color online) Same as Fig. 10 but for $k=8,9,10$ and $\beta^{\prime}=12,16,17$.

\begin{tabular}{|c|c|c|c|c|}
\hline$k$ & $\begin{array}{c}m=2 \\
\beta^{\prime}\end{array}$ & $\begin{array}{c}m=3 \\
\beta^{\prime}\end{array}$ & $\begin{array}{c}m=4 \\
\beta^{\prime}\end{array}$ & $\begin{array}{c}m=5 \\
\beta^{\prime}\end{array}$ \\
\hline 1 & 0 & 0 & 0 & 0 \\
2 & 3 & 1.5 & 1 & 0.75 \\
3 & 6 & 4 & 3 & 2 \\
4 & 11 & 7 & 6 & 4 \\
5 & 15 & 11 & 8 & 7 \\
6 & 22 & 15 & 11 & 10 \\
7 & 28 & 20 & 15 & 13 \\
8 & 36 & 25 & 20 & 16 \\
9 & 43 & 31 & 24 & 20 \\
10 & 55 & 37 & 29 & 24 \\
11 & 64 & 44 & 34 & 28 \\
12 & 75 & 51 & 40 & 33 \\
13 & & 59 & & \\
14 & & 67 & & \\
15 & & 76 & & \\
16 & & 85 & & \\
\hline
\end{tabular}

TABLE III: Tabulation of higher-order indices $\beta^{\prime}$ for various $k$ and superposition of $m$ CUEs, each having dimension $N=$ 10000 . 
and

$$
\beta^{\prime}=1+k+\frac{5(k-3)}{2}+\frac{(k-3)(k-5)}{4}, k=3,5,7, \ldots
$$

By suitable change of variables as $l=k / 2$ the Eq. 12 reduces to $\beta^{\prime}=l^{2}+3 l-3$ where $l=1,2,3, \ldots$. While using $q=(k-1) / 2$ the Eq. 13 reduces to $\beta^{\prime}=q^{2}+4 q-1$ where $q=1,2,3 \ldots$. Comparing these series with Eq. (3) one can see that it does not reduce to Eq. (3) for any $\beta$. Thus, using the scaling relations in Eqs. (12) and (13) no statement can be made at the level of jpdf of circular $\beta$-ensemble.

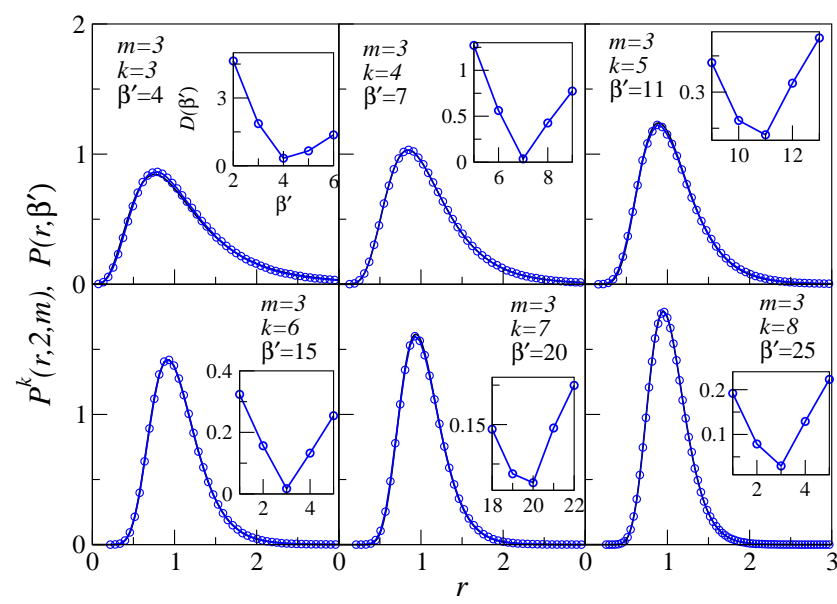

FIG. 12: (Color online) Distribution of the $k$-th (3 to 8) order spacing ratios (circles) for a superposition of $m=3$ CUE spectra. The dimension of the matrices is $N=10000$. The solid curve corresponds to $P\left(r, \beta^{\prime}\right)$ as given in Eq.(1) with $\beta^{\prime}$ given in Table III The insets shows $D$ as a function of $\beta^{\prime}$.

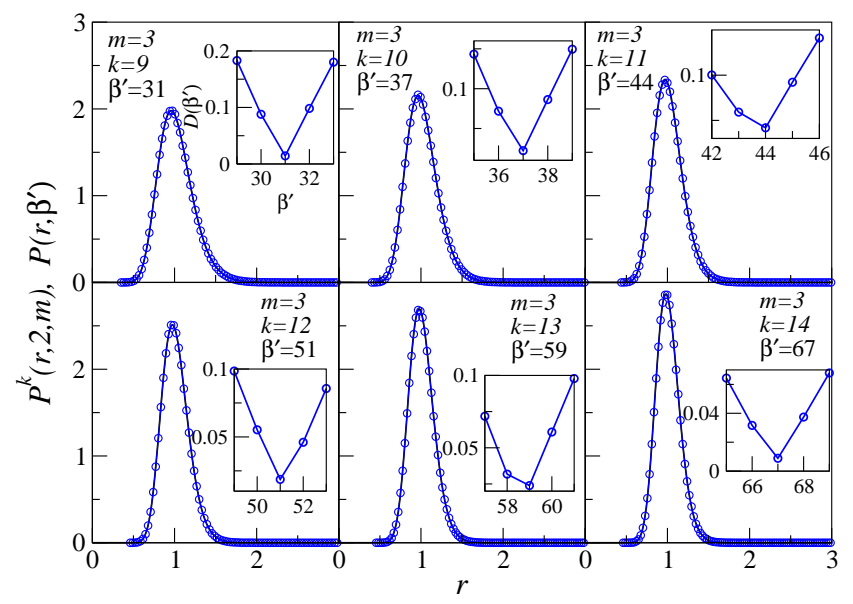

FIG. 13: (Color online) Same as Fig. 12 but for $k=9$ to 14 with corresponding $\beta^{\prime}$ given in Table III

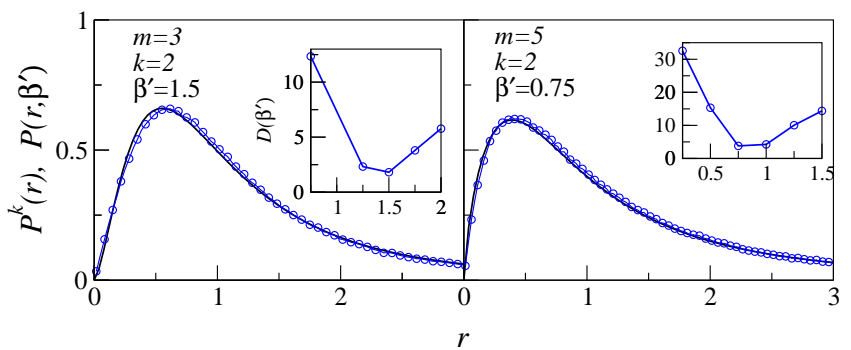

FIG. 14: (Color online) Same as Fig. 12 but for $k=2, m=3$ and 5 with corresponding noninteger values of $\beta^{\prime}$ given in Table III.

\section{SUPERPOSITION AND HIGHER-ORDER SPACING RATIOS IN CSE}

In this section, we study higher-order spacings ratio in the superposition of CSEs on the lines of Section III The CSE is used in modeling Hamiltonians with time-reversal symmetry and half-integer spin interaction [8, 112]. The results are tabulated in Table IV for $m=2$ to 6 and various values of $k$. The results are plotted in Fig. 15 and 16. In this case also except few all values of $\beta^{\prime}$ are whole number.

Based on results in Table IV we have given two conjectures at the level of spectral fluctuations. The first conjecture is as follows:

$$
P^{k}(r, 4, m)=P\left(r, \beta^{\prime}\right), \text { for } \beta^{\prime}=2 k+1=2 m+3
$$

and $m \geq 2$, while the second one is as follows:

$$
P^{k}(r, 4, m)=P\left(r, \beta^{\prime}\right), \text { for } \beta^{\prime}=2(k-1)=2(m-2)
$$

and $m \geq 3$. Our conjectures hold true for asymptotic value of $N$.

Although we are able to find scaling relations only for few cases, but for given $m$ one can compare the sequence of $\beta^{\prime}$ as a function of $k$ with that of $m^{\prime} \neq m$, within and across the Tables [I, III and IV It can be seen that these sequences are unique for given $m$ and the type of ensemble considered. One can also see that this is an increasing sequence on the lines of earlier result in $\mathrm{Eq} 3$ from Refs. 79, 80]. With this observation, we would like to conjecture that for a given number of symmetries $m$ and the Dyson index $\beta$ of the circular ensemble or a quantum chaotic system, the sequence of $\beta^{\prime}$ is an increasing function of $k$ and completely characterize the ensemble or the system uniquely.

Similarly, for given $k$ one can compare the sequence of $\beta^{\prime}$ as a function of $m$ with that of $k^{\prime} \neq k$, within and across the Tables [II [II and IV. Also, the sequences are unique for given $k$ and decreasing. Thus, with this observation, we would like to conjecture that for given $k$ and the Dyson index $\beta$, the sequence of $\beta^{\prime}$ is decreasing as a function of $m$ and is unique. This can be interpreted physically as follows: The level repulsion present in the 


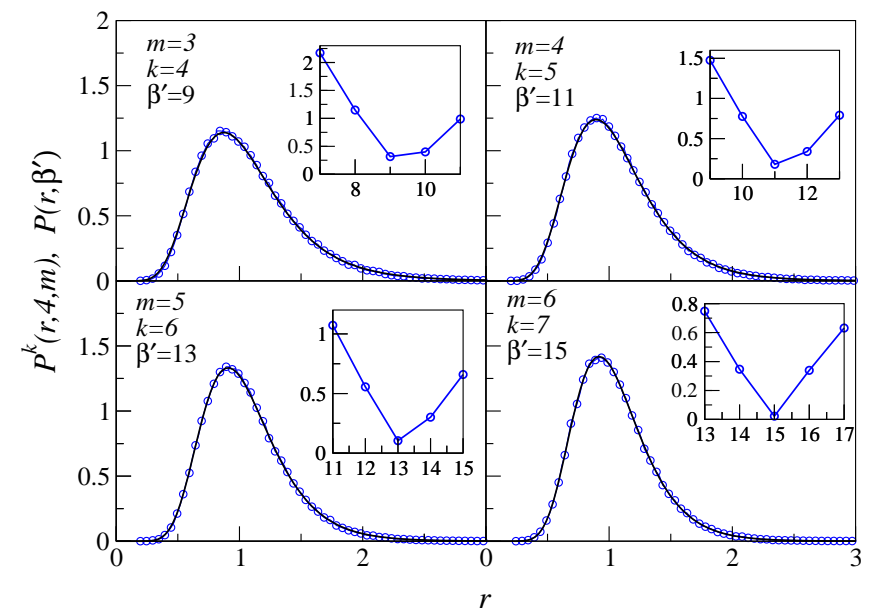

FIG. 15: (Color online) Distribution of the $k$-th order spacing ratios (circles) for various values of $k$ and superposition of $m$ CSE spectra. The dimension of the matrices is $N=1000$. The solid curve corresponds to $P\left(r, \beta^{\prime}\right)$ as given in Eq. (1) with $\beta^{\prime}$ given in Table IV The insets shows $D$ as a function of $\beta^{\prime}$.

eigenvalues characterized by $\beta^{\prime}$ for given $m$ and $k$ is reduced as $m$ is increased and is reflected in the reduction of the new value of $\beta^{\prime}$. The implication of this conjecture is that for given $\beta, k$ and $m \rightarrow \infty$ we will see $\beta^{\prime} \rightarrow 0$. It can be seen easily that one conjecture does not imply the second one.

Proving our results mathematically is challenging but we give an intuitive argument for the last conjecture. We know that NN as well as the $k$-th eigenvalues in circular or Gaussian spectra repel each other [7]. It has also been established mathematically (analytical and numerical) that when two same dimensional COE/GOE spectra are superposed the NN don't repel each other (level clustering) in the limit of matrix dimensions tending to infinity, which results in their spacings distribution to be Poissonian [8, 9, 46 48]. In other words, before superposition, the level repulsion present (characterized by $\beta=1$ ) has now vanished after superposition (characterized by $\beta=0)$. And this is what is also observed in our last conjecture i.e. for given $k$ and $m$ the value of $\beta^{\prime}$ (characterizing the repulsion between the $k$-th eigenvalues) reduces as $m$ is increased. In most of the cases we studied here, although not guaranteed, these reductions are integer number. The special case of our conjecture where $k=1, N=2$ and $m \rightarrow \infty$ is shown to have NN spacing distribution as Poissonian in Ref. [46].

\section{NUMERICAL METHODS}

Now, various numerical pieces of evidence supporting our results are presented. These best fits are checked with the numerical data quantitatively.

As a numerical check for our claims, analysis using

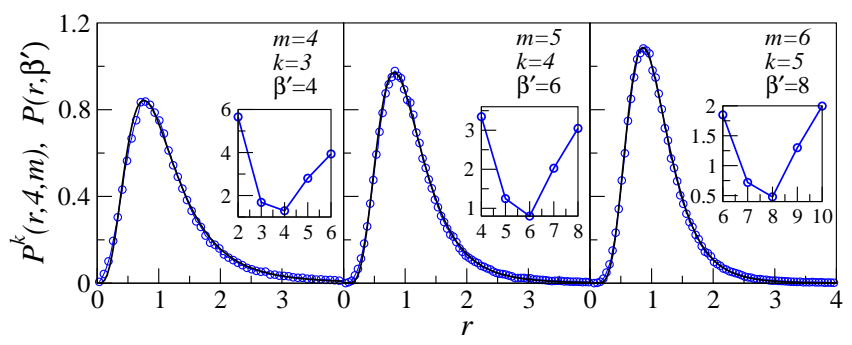

FIG. 16: (Color online) Same as Fig. 15 but for different values of $k$ and $m$.

\begin{tabular}{|c|c|c|c|c|c|}
\hline$k$ & $\begin{array}{c}m=2 \\
\beta^{\prime}\end{array}$ & $\begin{array}{c}m=3 \\
\beta^{\prime}\end{array}$ & $\begin{array}{c}m=4 \\
\beta^{\prime}\end{array}$ & $\begin{array}{c}m=5 \\
\beta^{\prime}\end{array}$ & $\begin{array}{c}m=6 \\
\beta^{\prime}\end{array}$ \\
\hline 1 & 0 & 0 & 0 & 0 & 0 \\
2 & 5 & 2 & 1 & 0.75 & 0.75 \\
3 & 7 & 7 & 4 & 2.5 & 2 \\
4 & 18 & 9 & 8 & 6 & 4 \\
5 & 21 & 14 & 11 & 10 & 8 \\
6 & 36 & 23 & 14 & 13 & 12 \\
7 & 39 & 27 & 21 & 16 & 15 \\
8 & 60 & 35 & 28 & 20 & 18 \\
9 & 63 & 47 & 33 & 27 & 21 \\
10 & 88 & 53 & 38 & 35 & 27 \\
11 & 92 & 63 & 48 & 40 & 34 \\
12 & 122 & 79 & 59 & 44 & 40 \\
\hline
\end{tabular}

TABLE IV: Tabulation of higher-order indices $\beta^{\prime}$ for various $k$ and superposition of $m$ CSEs each having dimension $N=$ 1000 .

Eq. (3) for $P\left(r, \beta^{\prime}\right)$ is carried, where no fitting parameter is involved. For this, the difference between the cumulative distributions is numerically found and defined as follows:

$$
D\left(\beta^{\prime}\right)=\sum_{i}\left|F_{\mathrm{obs}}^{k}\left(r_{i}, \beta, m\right)-F\left(r_{i}, \beta^{\prime}\right)\right|,
$$

where $F_{\text {obs }}^{k}(r, \beta, m)$ and $F\left(r, \beta^{\prime}\right)$ denotes cumulative distribution functions corresponding to the observed histogram $P_{\text {obs }}^{k}(r, 1, m)$ and the numerical fit or the postulated function $P\left(r, \beta^{\prime}\right)$ respectively. This definition has been used in earlier works [21, 79, 80] in similar kind of analysis. It can be seen that, $D\left(\beta^{\prime}\right)$ can take any positive value (upper bound) depending on the range of $i$ in the summation, but is minimum only for that value of $\beta^{\prime}$ for which $P\left(r, \beta^{\prime}\right)$ is best fit for the observed histogram. The values of $k$ for given $m$ are same as that in Figs. 1, 2, 3 , 4, 5, 6, 7, 8, 9, 10, 11, 12, 13, 15, and 16, The results of $D\left(\beta^{\prime}\right)$ are shown in the insets of these figures. It can be seen that the minima of $D\left(\beta^{\prime}\right)$ in each case coincides remarkably with that of corresponding $\beta^{\prime}$ from the main figures.

After finding the best fit for the observed data using $D\left(\beta^{\prime}\right)$, we go on to check how close the two probability distributions and their respective cumulative fuctions are. Firstly, the overlap $(p)$ between the probability plots 
in Figs. 1, 2, 3, 4, 5, 6, 7, 8, 9, 10, 11, 12, 13, 15, and 16 is calculated using the following definition:

$$
p=1-\int\left|P_{\mathrm{obs}}^{k}(r, \beta, m)-P\left(r, \beta^{\prime}\right)\right| d r .
$$

Secondly, the cumulative distribution functions corresponding to observed data $P_{\mathrm{obs}}^{k}(r, \beta, m)$ and $P\left(r, \beta^{\prime}\right)$ is studied. The maximum absolute difference $(d)$ between these cumulative distributions is calculated using the following definition:

$$
d=\operatorname{Sup}_{r_{i}}\left|F_{\mathrm{obs}}^{k}\left(r_{i}, \beta, m\right)-F\left(r_{i}, \beta^{\prime}\right)\right| .
$$

By definition $0 \leq p, d \leq 1$ and larger (smaller) value of $p(d)$ will indicate that the numerically observed distribution is close to that of the postulated one. Unlike $D\left(\beta^{\prime}\right)$, these values will only improve as the range of $i$ is increased. The values are shown in Tables V VI. VII] VIII, IX, X] and XI. The Tables V, VI, VII and VIII gives strong evidences for our results in Tables II] III and IV corresponding to the superposition of COE, CUE and CSE respectively. In Tables $[\mathrm{IX}, \mathrm{X}$ and $\mathrm{XI}$ results are shown for the physical system of QKT, measured nuclear resonances and the spin Hamiltonian as plotted in Figs 6 . 17. 8, 9, 10, and 11. It can be seen that in all the cases the values of $p$ and $d$ shown in these tables gives strong evidences for our results in these figures. The effect of small sample size in the case of nuclear resonances can be seen in the Table $\mathrm{X}$.

\begin{tabular}{|c|c||c|c||c|c|}
\hline$k$ & $\begin{array}{c}m=2 \\
p, d\end{array}$ & $k$ & $\begin{array}{c}m=3 \\
p, d\end{array}$ & $k$ & $m=4$ \\
& & $p, d$ \\
\hline 3 & $0.983,0.004153$ & 3 & $0.992,0.00085$ & 2 & $0.991,0.00164$ \\
5 & $0.986,0.003421$ & 4 & $0.991,0.00117$ & 4 & $0.991,0.00156$ \\
7 & $0.989,0.002506$ & 6 & $0.994,0.00057$ & 5 & $0.992,0.00116$ \\
9 & $0.994,0.001173$ & 7 & $0.989,0.00246$ & 6 & $0.995,0.00081$ \\
11 & $0.996,0.000651$ & 8 & $0.988,0.00268$ & 7 & $0.988,0.003263$ \\
13 & $0.993,0.001836$ & 9 & $0.994,0.00105$ & 8 & $0.995,0.00079$ \\
& & 10 & $0.988,0.0023$ & 9 & $0.986,0.003078$ \\
& & 11 & $0.995,0.0014$ & 11 & $0.996,0.000431$ \\
& & 12 & $0.995,0.00077$ & 13 & $0.994,0.001768$ \\
& & 13 & $0.994,0.00077$ & 15 & $0.996,0.000466$ \\
\hline
\end{tabular}

TABLE V: The overlap probability $p$ and the maximum absolute difference $d$ for the results on the superposition of COEs for $m=2,3,4$ and various $k$ 's.

\section{SUMMARY AND CONCLUSIONS}

This paper has studied the long-range correlations in the superposed spectra of COE, CUE and CSE using higher-order spacing ratios. We have given a table for the

\begin{tabular}{|c|c||c|c||c|c|}
\hline$k$ & $\begin{array}{c}m=5 \\
p, d\end{array}$ & $k$ & $\begin{array}{c}m=6 \\
p, d\end{array}$ & $k$ & $\begin{array}{c}m=7 \\
p, d\end{array}$ \\
\hline 2 & $0.957,0.01088$ & 3 & $0.967,0.00822$ & 3 & $0.941,0.001464$ \\
3 & $0.989,0.00186$ & 4 & $0.991,0.0009$ & 4 & $0.978,0.005134$ \\
6 & $0.995,0.00044$ & 5 & $0.965,0.00882$ & 5 & $0.991,0.000112$ \\
7 & $0.987,0.00253$ & 6 & $0.976,0.00562$ & 6 & $0.978,0.005358$ \\
8 & $0.981,0.00479$ & 7 & $0.988,0.00248$ & 7 & $0.974,0.006416$ \\
9 & $0.996,0.00046$ & 8 & $0.996,0.00046$ & 8 & $0.987,0.003019$ \\
10 & $0.990,0.00232$ & 9 & $0.983,0.00416$ & 9 & $0.990,0.002234$ \\
11 & $0.990,0.00264$ & 10 & $0.991,0.00241$ & 10 & $0.995,0.00096$ \\
12 & $0.996,0.00206$ & 12 & $0.996,0.00054$ & 15 & $0.997,0.00094$ \\
13 & $0.996,0.00037$ & 16 & $0.996,0.00138$ & 20 & $0.996,0.0013$ \\
\hline
\end{tabular}

TABLE VI: Same as Table V but for $m=5,6$ and 7 .

\begin{tabular}{|c|c||c|c|}
\hline$k$ & \multicolumn{1}{|c|}{$m=3$} & $k$ & \multicolumn{1}{c|}{$m=3$} \\
& \multicolumn{1}{|c|}{$p, d$} & & $p, d$ \\
\hline 3 & $0.9673,0.008161$ & 9 & $0.9977,0.0006759$ \\
4 & $0.9965,0.0007662$ & 10 & $0.9976,0.00080449$ \\
5 & $0.9813,0.005530$ & 11 & $0.9965,0.0015069$ \\
6 & $0.9948,0.000936$ & 12 & $0.9961,0.0005231$ \\
7 & $0.9893,0.004329$ & 13 & $0.9971,0.0011679$ \\
8 & $0.9960,0.0017572$ & 14 & $0.99709,0.0006004$ \\
\hline
\end{tabular}

TABLE VII: Same as Table $\square$ but for CUE and $m=3$.

\begin{tabular}{|c|c||c|c||c|c|}
\hline$k$ & $\begin{array}{c}m=4 \\
p, d\end{array}$ & $k$ & $\begin{array}{c}m=5 \\
p, d\end{array}$ & $k$ & $\begin{array}{c}m=6 \\
p, d\end{array}$ \\
\hline 2 & $0.9821,0.00884$ & 4 & $0.9966,0.00657$ & 3 & $0.9698,0.00709$ \\
3 & $0.9538,0.009765$ & 5 & $0.9896,0.00114$ & 4 & $0.9530,0.00870$ \\
4 & $0.9784,0.005174$ & 6 & $0.9896,0.00216$ & 5 & $0.9760,0.00540$ \\
5 & $0.9856,0.00353$ & 7 & $0.9880,0.00209$ & 6 & $0.9850,0.00379$ \\
6 & $0.988,0.002077$ & 8 & $0.9888,0.00247$ & 7 & $0.9918,0.00129$ \\
7 & $0.9907,0.002545$ & 9 & $0.9872,0.00272$ & 8 & $0.9874,0.00298$ \\
8 & $0.9873,0.002881$ & 10 & $0.9948,0.00132$ & 9 & $0.9931,0.00100$ \\
9 & $0.9913,0.002375$ & 11 & $0.9941,0.00114$ & 10 & $0.9916,0.00195$ \\
10 & $0.9917,0.001246$ & 12 & $0.9923,0.00152$ & 11 & $0.9907,0.00179$ \\
11 & $0.9938,0.001067$ & & & 12 & $0.9897,0.00212$ \\
12 & $0.9636,0.001533$ & & & & \\
\hline
\end{tabular}

TABLE VIII: Same as Table $\mathrm{V}$ but for CSE and $m=4,5$ and 6 .

\begin{tabular}{|c|c|c|c|c|c|}
\hline$k$ & $\beta^{\prime}$ & $p, d$ & $k$ & $\beta^{\prime}$ & $p, d$ \\
\hline 2 & 2 & $0.97263,0.003742$ & 8 & 23 & $0.97814,0.002949$ \\
3 & 4 & $0.95997,0.008608$ & 9 & 28 & $0.98059,0.002558$ \\
4 & 7 & $0.97056,0.003950$ & 10 & 34 & $0.97707,0.002847$ \\
5 & 10 & $0.97393,0.004558$ & 11 & 40 & $0.97981,0.001904$ \\
6 & 14 & $0.98118,0.001842$ & 12 & 47 & $0.97895,0.004052$ \\
7 & 18 & $0.97883,0.003812$ & 13 & 54 & $0.98250,0.002808$ \\
\hline
\end{tabular}

TABLE IX: The overlap probability $p$ and the maximum absolute difference $d$ for the distribution of higher-order spacing ratios using the eigenvalues of QKT in chaotic case. The value of $\beta^{\prime}$ for given $k$ is same as that in Fig. 6 and 7.

modified Dyson indices $\left(\beta^{\prime}\right)$ corresponding to the distribution of $k$-th order spacing ratio when $m$ number of ma- 


\begin{tabular}{|c|c|c||c|c|c|}
\hline$k$ & $\beta^{\prime}$ & $p, d$ & $k$ & $\beta^{\prime}$ & $p, d$ \\
\hline 2 & 2 & $0.88868,0.018197$ & 4 & 6 & $0.89450,0.028502$ \\
3 & 4 & $0.85402,0.027265$ & 5 & 8 & $0.87177,0.031754$ \\
\hline
\end{tabular}

TABLE X: Same as Table IX but for the data of nuclear resonances. The value of $\beta^{\prime}$ for given $k$ is same as that in Fig. 8

\begin{tabular}{|c|c|c|c|c|c|}
\hline$k$ & $\beta^{\prime}$ & $p, d$ & $k$ & $\beta^{\prime}$ & $p, d$ \\
\hline 2 & 2 & $0.93226,0.01725$ & 2 & 1 & $0.92570,0.01059$ \\
3 & 4 & $0.92965,0.01293$ & 3 & 2.5 & $0.95498,0.01021$ \\
4 & 7 & $0.93976,0.01779$ & 4 & 4 & $0.95163,0.00550$ \\
5 & 11 & $0.92546,0.03858$ & 5 & 6 & $0.93557,0.01493$ \\
6 & 12 & $0.95894,0.00826$ & 6 & 8 & $0.93278,0.01389$ \\
7 & 16 & $0.94367,0.01096$ & 7 & 10 & $0.94717,0.01175$ \\
& & 8 & 12 & $0.94094,0.00822$ \\
& & 9 & 16 & $0.94468,0.01891$ \\
& & 10 & 17 & $0.94992,0.00791$ \\
\hline
\end{tabular}

TABLE XI: The overlap probability $p$ and the maximum absolute difference $d$ for the distribution of higher-order spacing ratios using the eigenvalues of spin chains in chaotic case. The value of $\beta^{\prime}$ for given $k$ is same as that in Figs. 9 (left), 10 (right) and 11 (right).

trices each from COEs, CUEs and CSEs are superposed. For the case when two COEs are superposed two scaling relations relating $\beta^{\prime}$ and $k$ are found for even and odd values of $k$ respectively. The relation corresponding to even $k$ is related to the earlier result on the connection between CUE and superposition of two COEs at the level of jpdf [22, 23]. Conjectures on the lines of Ref. 21] are given. For the case of COE, it is conjectured that for given $m$, the distribution of the $k$-th order spacing ratio is related to $\beta^{\prime}$ such that the relation $\beta^{\prime}=k+1=m+2$ for $m \geq 2$ and $\beta^{\prime}=k-1=m-4$ for $m \geq 5$ holds true. Similarly, for the case of CSE, the relation $\beta^{\prime}=2 k+1=2 m+3$ for $m \geq 2$ and $\beta^{\prime}=2(k-1)=2(m-2)$ for $m \geq 3$ holds true.

We have tested our results on three different physical systems. The first one is the QKT in the quantum chaotic limit belonging to COE. The other two are the measured nuclear resonances and a spin Hamiltonian both corresponding to the GOE. These systems are known to have symmetries. For the case of QKT, we have tested our RMT results of $m=2$ case up to $k=13$ and found very good agreement. This agrees with the earlier analytical result from the Ref. [19], where it is shown that its Hamiltonian has two symmetries. Whereas in the case of nuclear resonances we could find agreement only up to $k=3$ due to nonuniform density as well as small sample size. But the results of $k=2$ and 3 were enough to conclude the presence of two symmetries using the uniqueness of our tabulated COE results. The third system we considered is the quantum chaotic spin Hamiltonian. Depending on the values of the parameter a given spin sector can have two or four symmetries. In both the cases we tested our COE results of $m=2$ and $m=4$. In this case, also we observed effects of nonuniform density but at large values of $k$ compared to the previous case due to the large matrix dimension. These results imply that our RMT results hold very well to quantum chaotic physical systems modeled by circular ensembles. For others, we may see the deviations for higher values of $k$ depending on the system and its matrix dimension or the sample size. Despite this we could successfully find the symmetries of systems modeled by Gaussian ensembles. Looking at our results and the Refs. 7, 8, 21, 37 39 specially the Ref.[37] our results can be claimed to be true for Gaussian ensembles in the limit of large matrix dimensions. Thus, our results can be used to find the symmetries in unknown physical systems.

For the case of $m=3$ superposition of CUEs two scaling relations relating $\beta^{\prime}$ and $k$ are found for even and odd values of $k$. These scaling relations along with other results are confirmed numerically using large matrix dimensions. We have used various numerical tests for the verification of our results. We conjectured that for given $m(k)$, the sequence of $\beta^{\prime}$ as a function of $k(m)$ is increasing (decreasing) and is unique to a given circular $\beta$-ensemble. As a corollary, finding symmetries as well as whether a given quantum chaotic system is time-reversal invariant (with or without the spin degree of freedom) or not can be found unambiguously. The Gaussian ensembles have been implemented in various experimental systems [113 117]. Thus, our circular ensemble results can be tested using these experiments by taking experimental systems with suitable geometrical symmetry corresponding to given $m$.

This work has given rise to new future directions as well. We would like to test our results as an additional and stringent test for finding symmetries in various other quantum complex systems [21, 25, 118]. Various quantum chaotic systems with and without time-reversal invariance and having additional symmetries can be tested. Our study can be extended to the case when matrices of unequal dimensions are superposed which will be relevant to understand symmetries in various other spin systems [21, 25, 119, 120]. Our study can be extended to other relevant ensembles from RMT, for example the ensembles with chiral symmetry [121 127] and Wishart ensemble [4, 80, 128 130].

\section{ACKNOWLEDGMENTS}

Authors are grateful to M. S. Santhanam, Harshini Tekur and Ravi Prakash for useful comments and discussions at various stages of this paper. UTB thanks Rukmani Bai and Hrushikesh Sable for helping in the numerical data. The results presented in the paper are based on the computations using Mathematica 9 in Vikram100, the 100TFLOP HPC Cluster at Physical Research Laboratory, Ahmedabad, India. 
[1] H. Weidenmüller and G. Mitchell, Reviews of Modern Physics 81, 539 (2009).

[2] G. E. Mitchell, A. Richter, and H. A. Weidenmüller, Reviews of Modern Physics 82, 2845 (2010).

[3] F. Haake, Quantum Signatures of Chaos (Springer, 3rd Edition, Berlin, 2010).

[4] G. Akemann, J. Baik, and P. Di Francesco, The Oxford handbook of random matrix theory (Oxford University Press, 2011).

[5] H.-J. Stöckmann, Quantum Chaos: An Introduction (University Press, Cambridge, 1999).

[6] C. E. Porter, Statistical Theories of Spectra: Fluctuations (Academic Press, New York, 1965).

[7] P. J. Forrester, Log-Gases and Random Matrices (Princeton University Press, Princeton and Oxford, 2010).

[8] M. L. Mehta, Random Matrices (Elsevier Academic Press, 3rd Edition, London, 2004).

[9] T. Guhr, A. Müeller-Groeling, and H. A. Weidenmüeller, Phys. Rep. 299, 189 (1998).

[10] J. Hutchinson, J. Keating, and F. Mezzadri, Physical Review E 92, 032106 (2015).

[11] H. J. Wells, arXiv preprint arXiv:1410.1666 (2014).

[12] W.-J. Rao, Physical Review B 102, 054202 (2020).

[13] W.-J. Rao, Journal of Physics A: Mathematical and Theoretical 54, 105001 (2021).

[14] W.-J. Rao, arXiv preprint arXiv:2105.04468 (2021).

[15] J. Gómez, K. Kar, V. Kota, R. A. Molina, A. Relaño, and J. Retamosa, Physics Reports 499, 103 (2011).

[16] O. Bohigas, M. J. Giannoni, and C. Schmit, Phys. Rev. Lett. 52, 1 (1984).

[17] N. E. Hurt, Quantum chaos and mesoscopic systems: mathematical methods in the quantum signatures of chaos, vol. 397 (Springer Science \& Business Media, 2013).

[18] V. Oganesyan and D. A. Huse, Phys. Rev. B 75, 155111 (2007).

[19] F. Haake, M. Kus, and R. Scharf, Z. Phys. B 65, 381 (1987).

[20] L. E. Reichl, The Transition to Chaos, 2nd edition (Springer-Verlag, New York, 2004).

[21] S. H. Tekur and M. S. Santhanam, Phys. Rev. Research 2, 032063 (2020).

[22] F. J. Dyson, J. Math. Phys. 3, 166 (1962).

[23] J. Gunson, Journal of Mathematical Physics 3, 752 (1962).

[24] G. W. Anderson, A. Guionnet, and O. Zeitouni, An introduction to random matrices, vol. 118 (Cambridge university press, 2010).

[25] O. Giraud, N. Macé, E. Vernier, and F. Alet, arXiv preprint arXiv:2008.11173 (2020).

[26] L. F. Santos and M. Rigol, Phys. Rev. E 82, 031130 (2010).

[27] A. Gubin and L. F. Santos, Am. J. Phys. 80, 246 (2012).

[28] K. Brading and E. Castellani, Symmetries in physics: philosophical reflections (Cambridge University Press, 2003).

[29] S. Corry, Symmetry and Quantum Mechanics (Chapman and Hall/CRC, 2016).

[30] D. J. Gross, Proceedings of the National Academy of Sciences 93, 14256 (1996).
[31] J. Rosen, Symmetry rules: How science and nature are founded on symmetry (Springer Science \& Business Media, 2008).

[32] K. Tapp, Symmetry: a mathematical exploration (Springer Science \& Business Media, 2011).

[33] G. Longo and M. Montévil, Perspectives on organisms: Biological time, symmetries and singularities (Springer Science \& Business Media, 2013).

[34] G. Sardanashvily, Noether's Theorems (Springer, 2016).

[35] Y. Kosmann-Schwarzbach, in The Noether Theorems (Springer, 2011), pp. 55-64.

[36] J. S. Cotler, G. Gur-Ari, M. Hanada, J. Polchinski, P. Saad, S. H. Shenker, D. Stanford, A. Streicher, and M. Tezuka, Journal of High Energy Physics 2017, 118 (2017).

[37] S. H. Tekur, U. T. Bhosale, and M. Santhanam, Phys. Rev. B 98, 104305 (2018).

[38] C. E. Porter, Nuclear Physics 40, 167 (1963).

[39] P. B. Kahn and C. E. Porter, Nuclear Physics 48, 385 (1963).

[40] I. Dumitriu and A. Edelman, Journal of Mathematical Physics 43, 5830 (2002).

[41] W. Buijsman, V. Cheianov, and V. Gritsev, Phys. Rev. Lett. 122, 180601 (2019).

[42] P. Sierant and J. Zakrzewski, Phys. Rev. B 99, 104205 (2019).

[43] M. V. Berry, Proc. R. Soc. A 400, 229 (1985).

[44] M. Sieber and K. Richter, Physica Scripta 2001, 128 (2001).

[45] S. Müller, S. Heusler, A. Altland, P. Braun, and F. Haake, New Journal of Physics 11, 103025 (2009).

[46] T. Tkocz, M. Smaczyński, M. Kuś, O. Zeitouni, and K. Życzkowski, Random Matrices: Theor. Appl. 1, 1250009 (2012).

[47] M. Smaczyński, T. Tkocz, M. Kuś, and K. Życzkowski, Phys. Rev. E 88, 052902 (2013).

[48] T. Tkocz et al., Electronic communications in probability 18 (2013).

[49] M. V. Berry and M. Tabor, Proc. R. Soc. Lond. A 356, 375 (1977).

[50] F. J. Dyson, J. Math. Phys. 3, 140 (1962).

[51] F. Mezzadri, Notices of the AMS 54, 592 (2007).

[52] R. Killip and I. Nenciu, International Mathematics Research Notices 2004, 2665 (2004).

[53] M. L. Mehta and F. J. Dyson, J. Math. Phys. 4, 713 (1963).

[54] H. Bruus and J.-C. Angl'es d'Auriac, Phys. Rev. B 55, 9142 (1997).

[55] J. M. G. Gómez, R. A. Molina, A. Relaño, and J. Retamosa, Phys. Rev. E 66, 036209 (2002).

[56] V. Oganesyan and D. A. Huse, Phys. Rev. B 75, 155111 (2007).

[57] Y. Y. Atas, E. Bogomolny, O. Giraud, and G. Roux, Phys. Rev. Lett. 110, 084101 (2013).

[58] Y. Atas, E. Bogomolny, O. Giraud, P. Vivo, and E. Vivo, Journal of Physics A: Mathematical and Theoretical 46, 355204 (2013).

[59] V. Oganesyan, A. Pal, and D. A. Huse, Phys. Rev. B 80, 115104 (2009).

[60] A. Pal and D. A. Huse, Phys. Rev. B 82, 174411 (2010).

[61] S. Iyer, V. Oganesyan, G. Refael, and D. A. Huse, Phys. 
Rev. B 87, 134202 (2013)

[62] E. Cuevas, M. Feigel'Man, L. Ioffe, and M. Mezard, Nature communications 3, 1128 (2012).

[63] G. Biroli, A. Ribeiro-Teixeira, and M. Tarzia, arXiv preprint arXiv:1211.7334 (2012).

[64] C. Chen, F. Burnell, and A. Chandran, Phys. Rev. Lett. 121, 085701 (2018).

[65] L. F. Santos and M. Rigol, Phys. Rev. E 81, 036206 (2010).

[66] C. Kollath, G. Roux, G. Biroli, and A. M. Läuchli, Journal of Statistical Mechanics: Theory and Experiment 2010, P08011 (2010)

[67] M. Rigol and L. F. Santos, Phys. Rev. A 82, 011604 (2010).

[68] M. Collura, H. Aufderheide, G. Roux, and D. Karevski, Phys. Rev. A 86, 013615 (2012).

[69] E. Tarquini, G. Biroli, and M. Tarzia, Phys. Rev. Lett. 116, 010601 (2016)

[70] A. L. Corps and A. Relaño, Phys. Rev. E 101, 022222 (2020).

[71] F. Sun and J. Ye, Phys. Rev. Lett. 124, 244101 (2020).

[72] F. Sun, Y. Yi-Xiang, J. Ye, and W.-M. Liu, Phys. Rev. D 101, 026009 (2020).

[73] T. Nosaka and T. Numasawa, Journal of High Energy Physics 2020, 1 (2020).

[74] M. Srdinsek, T. Prosen, and S. Sotiriadis, arXiv preprint arXiv:2012.08505 (2020).

[75] N. Chavda, H. Deota, and V. Kota, Physics Letters A 378, 3012 (2014).

[76] V. Kota and N. Chavda, International Journal of Modern Physics E 27, 1830001 (2018).

[77] S. H. Tekur, S. Kumar, and M. S. Santhanam, Phys. Rev. E 97, 062212 (2018).

[78] L. Sá, P. Ribeiro, and T. Prosen, Phys. Rev. X 10, 021019 (2020).

[79] S. H. Tekur, U. T. Bhosale, and M. S. Santhanam, Phys. Rev. B 98, 104305 (2018).

[80] U. T. Bhosale, S. H. Tekur, and M. S. Santhanam, Phys. Rev. E 98, 052133 (2018).

[81] P. Rao, M. Vyas, and N. D. Chavda, The European Physical Journal Special Topics 229, 2603 (2020).

[82] T. A. Brody, J. Flores, J. B. French, P. A. Mello, A. Pandey, and S. S. M. Wong, Rev. Mod. Phys. 53, 385 (1981)

[83] A. Y. Abul-Magd and M. H. Simbel, Phys. Rev. E 60, 5371 (1999)

[84] N. M. Katz and P. Sarnak, Bull. Am. Math. 31, 1 (1999).

[85] J. P. Keating and N. C. Snaith, J. Phys. A: Math. Gen. 36, 2859 (2003).

[86] W.-J. Rao, Phys. Rev. B 102, 054202 (2020).

[87] W.-J. Rao and M. Chen, arXiv preprint arXiv:2006.07774 (2020).

[88] P. J. Forrester and E. M. Rains, Probability theory and related fields 130, 518 (2004).

[89] P. J. Forrester, Communications in mathematical physics 285, 653 (2009).

[90] U. T. Bhosale, arXiv preprint arXiv:1905.02585 (2019).

[91] J. Zakrzewski and M. Kuś, Phys. Rev. Lett. 67, 2749 (1991).

[92] R. Alicki, D. Makowiec, and W. Miklaszewski, Phys. Rev. Lett. 77, 838 (1996).

[93] Y. S. Weinstein, S. Lloyd, and C. Tsallis, Phys. Rev. Lett. 89, 214101 (2002).
[94] R. Demkowicz-Dobrzański and M. Kuś, Phys. Rev. E 70, 066216 (2004).

[95] S. Chaudhury, A. Smith, B. E. Anderson, S. Ghose, and P. S. Jessen, Nature 461, 768 (2009).

[96] M. Lombardi and A. Matzkin, Phys. Rev. E 83, 016207 (2011).

[97] Z. Puchała, L. Pawela, and K. Życzkowski, Phys. Rev. A 93, 062112 (2016).

[98] C. Neill, P. Roushan, M. Fang, Y. Chen, M. Kolodrubetz, Z. Chen, A. Megrant, R. Barends, B. Campbell, B. Chiaro, et al., Nature Physics (2016).

[99] U. T. Bhosale and M. S. Santhanam, Phys. Rev. E 95, 012216 (2017).

[100] J. B. Ruebeck, J. Lin, and A. K. Pattanayak, Phys. Rev. E 95, 062222 (2017).

[101] U. T. Bhosale and M. S. Santhanam, Phys. Rev. E 98, 052228 (2018).

[102] V. R. Krithika, V. S. Anjusha, U. T. Bhosale, and T. S. Mahesh, Phys. Rev. E 99, 032219 (2019).

[103] E. J. Meier, J. Ang'ong'a, F. A. An, and B. Gadway, Phys. Rev. A 100, 013623 (2019).

[104] M. H. Muñoz Arias, P. M. Poggi, P. S. Jessen, and I. H. Deutsch, Phys. Rev. Lett. 124, 110503 (2020).

[105] T. Xu, T. Scaffidi, and X. Cao, Phys. Rev. Lett. 124, 140602 (2020).

[106] S. Kumar, Phys. Rev. A 102, 012405 (2020).

[107] P. A. Miller and S. Sarkar, Phys. Rev. E 60, 1542-1550 (1999).

[108] G. Hacken, R. Werbin, and J. Rainwater, Phys. Rev. C 17, 43 (1978).

[109] T. C. Hsu and J. C. Angle‘s d'Auriac, Phys. Rev. B 47, 14291 (1993).

[110] L. F. Santos, Journal of mathematical physics 50, 095211 (2009).

[111] K. Kudo and T. Deguchi, Journal of the Physical Society of Japan 74, 1992 (2005).

[112] K. Zyczkowski, in Chaos-The Interplay Between Stochastic and Deterministic Behaviour (Springer, 1995), pp. 565-571.

[113] H. Alt, H.-D. Gräf, T. Guhr, H. L. Harney, R. Hofferbert, H. Rehfeld, A. Richter, and P. Schardt, Phys. Rev. E 55, 6674 (1997)

[114] C. Dembowski, H.-D. Gräf, A. Heine, H. Rehfeld, A. Richter, and C. Schmit, Phys. Rev. E 62, R4516 (2000).

[115] R. Schäfer, M. Barth, F. Leyvraz, M. Müller, T. H. Seligman, and H.-J. Stöckmann, Phys. Rev. E 66, 016202 (2002).

[116] B. Dietz, T. Guhr, B. Gutkin, M. Miski-Oglu, and A. Richter, Phys. Rev. E 90, 022903 (2014).

[117] A. Rehemanjiang, M. Allgaier, C. H. Joyner, S. Müller, M. Sieber, U. Kuhl, and H.-J. Stöckmann, Phys. Rev. Lett. 117, 064101 (2016).

[118] M. Srdinšek, T. c. v. Prosen, and S. Sotiriadis, Phys. Rev. Lett. 126, 121602 (2021).

[119] K. Binder and A. P. Young, Reviews of Modern physics 58, 801 (1986).

[120] I. Amico, R. Fazio, and A. Osterloh, Rev. Mod. Phys. 80, 517 (2008).

[121] Y. V. Fyodorov and E. Strahov, Nuclear Physics B 647, 581 (2002).

[122] P. H. Damgaard, in Journal of Physics: Conference Series (IOP Publishing, 2011), vol. 287, p. 012004. 
[123] V. Kaymak, M. Kieburg, and T. Guhr, Journal of Physics A: Mathematical and Theoretical 47, 295201 (2014).

[124] C. W. J. Beenakker, Rev. Mod. Phys. 87, 1037 (2015).

[125] G. Akemann, Stochastic Processes and Random Matrices: Lecture Notes of the Les Houches Summer School 104, 228 (2017).

[126] T. Mondal and P. Shukla, Phys. Rev. E 102, 032131 (2020).
[127] A. Rehemanjiang, M. Richter, U. Kuhl, and H.-J. Stöckmann, Phys. Rev. Lett. 124, 116801 (2020).

[128] J. Wishart, Biometrika 20A, 32 (1928).

[129] S. N. Majumdar and M. Vergassola, Phys. Rev. Lett. 102, 060601 (2009).

[130] M. Fridman, R. Pugatch, M. Nixon, A. A. Friesem, and N. Davidson, Phys. Rev. E 85, 020101 (2012). 